\title{
A Characterization of the Classical States of the Quantum Harmonic Oscillator by Means of de Finetti's Theorem
}

\author{
A. Bach and A. Srivastav* \\ Institut für Theoretische Physik I, Westfälische Wilhelms-Universität Münster, \\ D-4400 Münster, Federal Republic of Germany
}

\begin{abstract}
Integral representations of statistical operators in terms of coherent states are derived by means of a quantum version of the Poisson limit of de Finetti's theorem.
\end{abstract}

\section{Introduction}

Integral representations of states of indistinguishable particles which are based on the quantum extension of de Finetti's theorem [1] have a characteristic structure which underlines the underlying invariance under permutations. These integral representations are mixtures of homogeneous product states.

This particular structure, however, is lost if the limit of an array of indistinguishable particles is considered. A state which arises as the limit of an array of homogeneous product states is not immediately identifiable as such a limit. Therefore an integral representation in terms of these states is not straightforwardly recognized as a consequence of de Finetti's theorem.

In [2] it has been shown that the quantum harmonic oscillator - or, more generally, a one-dimensional system described by the familiar creation and annihilation operators $a^{+}, a$-is obtained in particular states as a limit from an array of Bose-Einstein symmetric two-level systems (quantum Bernoulli process). By means of a quantum formulation of the Poisson limit theorem it is shown that the well known coherent states of quantum optics are limits of homogeneous product states defined on the array of two-level systems.

It is the aim of this note to show that the integral representations of statistical operators in terms of coherent states, which are extensively used in quantum optics, are in fact the result of a quantum version of the Poisson limit of de Finetti's theorem.

These integral representations are defined as follows. A c.o.n.s. of the Hilbert space $H$ under consideration is given by the eigenvectors $\Phi(k), k \in \mathbb{Z}_{+}$, of the number operator $a^{+} a$. For any $z \in \mathbb{C}$ a coherent state vector $\Psi(z) \in H$ is defined by, $z=(\lambda)^{1 / 2} \exp (i \alpha)$

$$
\Psi(z)=\sum_{k=0}^{\infty}\left\{\pi_{\lambda}(k)\right\}^{1 / 2} \exp (i \alpha k) \Phi(k),
$$

* Present address: Institut für Diskrete Mathematik, Universität Bonn, D-5300 Bonn, Federal Republic of Germany 
where for $\lambda \in \mathbb{R}_{+}, k \in \mathbb{Z}_{+}$,

$$
\pi_{\lambda}(k)=(k !)^{-1} \exp (-\lambda) \lambda^{k}
$$

denotes the Poisson distribution.

The statistical operator of a coherent state is the orthogonal projection defined by $\Psi(z), z \in \mathbb{C}$,

$$
P(z)=\langle\Psi(z), \cdot\rangle \Psi(z)
$$

A state is called a classical state if it can be identified with a statistical operator $W=W(\mu)$ which is defined by the integral representation

$$
W(\mu)=\int d \mu(z) P(z) .
$$

Here $\mu$ is a probability measure on $\mathbb{C}, \mu \in M_{+}^{1}(\mathbb{C})$, and the integral is a trace class operator valued Bochner integral. For the properties of classical states we refer to $[3,4]$.

To show that the integral representation (4) is a consequence of de Finetti's theorem we recall, in Sect. 2, some basic facts concerning integral representations of states of classical indistinghuishable particles. In Sect. 3 we reformulate the quantum de Finetti theorem of Hudson and Moody [1] for a system of BoseEinstein symmetric two-level systems. Following the strategy of [2] we build up the structure of a canonical pair by means of an array of two-level systems in Sect. 4. This array is used to derive the integral representation (4). We conclude with some remarks in Sect. 5.

\section{Indistinguishable Classical Particles}

In this section we are concerned with the familiar statistical setting where $n$ identical particles are distributed on two groups of cells. For the description we introduce a probability space $(\Omega, F, P)$ and random variables $X_{i}: \Omega \rightarrow\{0,1\}$, $1 \leqq i \leqq n$, where $\left[X_{i}=1\right]$ denotes the event that particle $i$ is in the first group of cells.

The particles are called indistinguishable if the random variables $X_{i}, 1 \leqq i \leqq n$, are interchangeable, i.e. if

$$
P\left(X_{i}=j_{i}, 1 \leqq i \leqq n\right)=P\left(X_{\pi(i)}=j_{i}, 1 \leqq i \leqq n\right)
$$

holds for any $\mathbf{j} \in\{0,1\}^{n}$ and any permutation $\pi$ of the integers $\{1, \ldots, n\}$. A countably infinite sequence of random variables $X_{i}, i \in \mathbb{N}$, is called interchangeable if any finite subset is interchangeable. For a review of the concept of interchangeability we refer to [5]. The concept of indistinguishable classical particles is discussed in [6].

For a countably infinite set of indistinguishalbe particles there exists an integral representation for the probability that from $n, n \in \mathbb{N}$, arbitrarily chosen particles there are exactly $k, 0 \leqq k \leqq n$, in the first group of cells. This integral representation is defined in terms of that probability distribution which characterizes i.i.d. particles, i.e. the binomial distribution, $n \in \mathbb{N}, 0 \leqq k \leqq n, p \in[0,1]$,

$$
B_{n, p}(k)=\left(\begin{array}{l}
n \\
k
\end{array}\right) p^{k}(1-p)^{n-k}
$$


Theorem 1 (de Finetti) (cf. [5, 7]). Assume that the random variables $X_{i}: \Omega \rightarrow\{0,1\}$, $i \in \mathbb{N}$, are interchangeable. Then there exists a uniquely determined probability measure $v \in M_{+}^{1}([0,1])$ such that for any $n \in \mathbb{N}$ and any $k, 0 \leqq k \leqq n$,

holds.

$$
P\left(\sum_{i=1}^{n} X_{i}=k\right)=\int d v(p) B_{n, p}(k)
$$

Example 1. If $n$ particles are distributed on $d$ cells according to Bose-Einstein statistics and one cell is arbitrarily chosen (this is group one) the probability for the event $[\mathbf{X}=\mathbf{j}], \mathbf{X}=\left(X_{1}, \ldots, X_{n}\right), \mathbf{j} \in\{0,1\}^{n}$, is given by

$$
P(\mathbf{X}=\mathbf{j})=1^{[k]}(d-1)^{[n-k]} / d^{[n]},
$$

where $k=\sum j_{i}$ and for $x \in \mathbb{R}, n \in \mathbb{N}$,

$$
x^{[n]}=x(x+1) \ldots(x+n-1) .
$$

The probability that group one contains just $k, 0 \leqq k \leqq n$, particles is therefore determined by a Polya distribution

$$
P\left(\sum_{i=1}^{n} X_{i}=k\right)=\left(\begin{array}{c}
d+n-1 \\
n
\end{array}\right)^{-1}\left(\begin{array}{c}
d+n-k-2 \\
n-k
\end{array}\right) .
$$

As the random variables defined by Eq. (8) are interchangeable and can be considered as the segment of a countably infinite sequence of interchangeable random variables de Finetti's theorem applies and we have for all $n \in \mathbb{N}$ and any $k$, $0 \leqq k \leqq n$,

$$
\left(\begin{array}{c}
d+n-1 \\
n
\end{array}\right)^{-1}\left(\begin{array}{c}
d+n-k-2 \\
n-k
\end{array}\right)=\int_{0}^{1} d p(d-1)(1-p)^{d-2} B_{n, p}(k) .
$$

In applications of the setting introduced at the beginning of this section one usually is interested in macroscopic quantities, i.e. in the situation when the number of particles is arbitrarily large. For obvious reasons we call the limit of the integral representation (7) the Poisson limit of de Finetti's theorem.

Theorem 2 [8]. Let be given for any $n \in \mathbb{N}$ random variables $X_{n, i}: \Omega_{n} \rightarrow\{0,1\}, i \in \mathbb{N}$, defined on a probability space $\left(\Omega_{n}, F_{n}, P_{n}\right)$ which are supposed to be interchangeable for any fixed $n$. Denote by $\mu_{n}, \mu_{n} \in M_{+}^{1}\left(\mathbb{R}_{+}\right)$, the image of the mixing measure $v_{n} \in M_{+}^{1}([0,1])$ associated with the interchangeable sequence $X_{n, i}$ ( $n$ fixed) under the mapping $T_{n}:[0,1] \rightarrow \mathbb{R}_{+}$defined by $T_{n}(p)=n p$. Moreover, assume that the sequence $\mu_{n}, n \in \mathbb{N}$, converges weakly to a probability measure $\mu \in M_{+}^{1}\left(\mathbb{R}_{+}\right)$then for any $k \in \mathbb{Z}_{+}$the integral representation

holds.

$$
\lim _{n \rightarrow \infty} P_{n}\left(\sum_{i=1}^{n} X_{n, i}=k\right)=\int d \mu(\lambda) \pi_{\lambda}(k)
$$

We remark that the theorem is due to Benczur [8]. A different proof is possible by means of Lemma 1 below (with the obvious modifications where $\mathbb{C}$ is replaced by $\mathbb{R}_{+}$) setting

$$
\begin{gathered}
f_{n}(\lambda)=B_{n, \lambda / n}(k) 1_{[0, n]}(\lambda), \\
f(\lambda)=\pi_{\lambda}(k) .
\end{gathered}
$$


Example 2. Generalizing Example 1 we assume that in any row of the interchangeable array $X_{n, i}$ the sequence $X_{n, i}(n$ fixed) is characterized by a beta distribution as mixing measure such that, $n \in \mathbb{N}$,

$$
\begin{aligned}
P_{n}\left(\sum_{i=1}^{n} X_{n, i}=k\right) & =\left(\begin{array}{c}
d(n)+n-1 \\
n
\end{array}\right)^{-1}\left(\begin{array}{c}
d(n)+n-k-2 \\
n-k
\end{array}\right) \\
& =\int_{0}^{1} d p(d(n)-1)(1-p)^{d(n)-2} B_{n, p}(k)
\end{aligned}
$$

holds where we assume that $d(n) \in \mathbb{N}$ satisfies $n / d(n) \rightarrow \bar{n} \in \mathbb{R}_{+}$for $n \rightarrow \infty$.

It is well known that under these conditions the Polya distribution converges to a geometric distribution with mean $\bar{n}$

$$
\lim _{n \rightarrow \infty}\left(\begin{array}{c}
d(n)+n-1 \\
n
\end{array}\right)^{-1}\left(\begin{array}{c}
d(n)+n-k-2 \\
n-k
\end{array}\right)=\frac{1}{1+\bar{n}}\left(\frac{\bar{n}}{1+\bar{n}}\right)^{k} .
$$

On the other hand the distribution function of the image of the beta distribution, $\lambda \in[0, n], n \in \mathbb{N}$,

$$
F_{n}(\lambda)=1-\left(1-\frac{\lambda}{n}\right)^{d(n)-1}
$$

converges to the distribution function of an exponential distribution with mean $\bar{n}$,

$$
\lim _{n \rightarrow \infty} F_{n}(\lambda)=1-\exp (-\lambda / \bar{n})
$$

Accordingly, we obtain the following integral representation of the geometric distribution (cf. [9]), $k \in \mathbb{Z}_{+}$,

$$
\frac{1}{1+\bar{n}}\left(\frac{\bar{n}}{1+\bar{n}}\right)^{k}=\int d \lambda(\bar{n})^{-1} \exp (-\lambda / \bar{n}) \pi_{\lambda}(k) .
$$

\section{The Quantum de Finetti Theorem for Two-Level Systems}

The quantum analogue of a random variable with values in $\{0,1\}$ is a two-level system (TLS) with Hilbert space $\mathbb{C}^{2}$. In $\mathbb{C}^{2}$ we fix an orthonormal basis $e_{1}, e_{2}$ describing the lower and the upper level, respectively. The algebra of observables of the system is the algebra $B\left(\mathbb{C}^{2}\right)$ of all complex $2 \times 2$ matrices.

A set of $n, n \in \mathbb{N}$, TLS is described by $\otimes^{n} B\left(\mathbb{C}^{2}\right)$. A state on $\otimes^{n} B\left(\mathbb{C}^{2}\right)$ is identified with a statistical operator $W_{n}$ defined on the $2^{n}$ dimensional Hilbert space $\stackrel{n}{\otimes}^{2}$. On this Hilbert space we introduce the usual permutation operators $U_{\pi}$ which are defined by their action on the basis

$$
\begin{gathered}
e_{i_{1}} \otimes e_{i_{2}} \otimes \ldots \otimes e_{i_{n}}, \quad i_{j} \in\{1,2\}, \quad 1 \leqq j \leqq n, \\
U_{\pi} \otimes_{j=1}^{n} e_{i_{j}}=\bigotimes_{j=1}^{n} e_{i_{\pi(j)}},
\end{gathered}
$$

and extended by linearity.

The $n$ TLS are called indistinguishable if the statistical operator $W_{n}$ is invariant under all permutations of the TLS, i.e. if

$$
U_{\pi} W_{n} U_{\pi}^{+}=W_{n}
$$


holds for all permutations $\pi$ of the integers $\{1, \ldots, n\}$. The $n$ TLS are called bosons if the statistical operator is Bose-Einstein symmetric, i.e. if $W_{n}$ satisfies the stronger symmetry condition that

$$
U_{\pi} W_{n}=W_{n}
$$

holds for all permutations $\pi$.

The orthogonal projection onto the Bose-Einstein symmetric subspace $\left(\stackrel{n}{\otimes} \mathbb{C}^{2}\right)_{+}$of $\stackrel{n}{\otimes} \mathbb{C}^{2}$ is defined by

$$
\Pi_{+}^{(n)}=(n !)^{-1} \sum_{\pi} U_{\pi} .
$$

If $W_{n}$ is Bose-Einstein symmetric we have

$$
W_{n}=W_{n} \Pi_{+}^{(n)}=\Pi_{+}^{(n)} W_{n}
$$

such that the definition of $W_{n}$ can be restricted to the Bose-Einstein symmetric subspace as is done usually.

A c.o.n.s. of $\left(\stackrel{n}{\otimes} \mathbb{C}^{2}\right)_{+}, \operatorname{dim}\left(\left(\stackrel{n}{\otimes} \mathbb{C}^{2}\right)_{+}\right)=n+1$, is given by the vectors, $0 \leqq k \leqq n$,

$$
\phi_{n}(k)=\left(\begin{array}{l}
n \\
k
\end{array}\right)^{1 / 2} \Pi_{+}^{(n)} \otimes e_{1}^{n-k} \otimes e_{2}^{k} .
$$

These vectors are called $n$-number state vectors. The homogeneous product vectors

$$
\psi_{n}(z)=\otimes^{n} \psi(z),
$$

where $z \in D, D=\left\{u \in \mathbb{C} ;|u|^{2} \leqq 1\right\}$, and

$$
\psi(z)=\left(1-|z|^{2}\right)^{1 / 2} e_{1}+z e_{2}
$$

are a total set in $\left(\stackrel{n}{\otimes} \mathbb{C}^{2}\right)+$ and are called $n$-coherent state vectors. The following formulae are obvious from the definitions, $z=|z| \exp (i \alpha)$,

$$
\begin{gathered}
\left\langle\phi_{n}(k), \psi_{n}(z)\right\rangle=\left\{B_{n,\left.|z|\right|^{2}}(k)\right\}^{1 / 2} \exp (i \alpha k), \\
\left\langle\psi_{n}\left(z^{\prime}\right), \psi_{n}(z)\right\rangle=\left[\bar{z}^{\prime} z+\left\{\left(1-\left|z^{\prime}\right|^{2}\right)\left(1-|z|^{2}\right)\right\}^{1 / 2}\right]^{n} .
\end{gathered}
$$

Up to a phase any normalized vector of $\mathbb{C}^{2}$ can be identified with a 1-coherent state vector. For any pure state on $B\left(\mathbb{C}^{2}\right)$ there exists therefore a $z \in D$ such that

$$
W_{1}=\langle\psi(z), \cdot\rangle \psi(z)
$$

holds. In Eq. (30) $z \in D$ is uniquely determined up to the exceptional case where $|z|=1$, i.e. for

$$
W_{1}=\left\langle e_{2}, \cdot\right\rangle e_{2} .
$$

It follows that any homogeneous product state of pure states on $\stackrel{n}{\otimes} B\left(\mathbb{C}^{2}\right)$ is obviously Bose-Einstein symmetric and is determined by a projection

$$
P_{n}(z)=\left\langle\psi_{n}(z), \cdot\right\rangle \psi_{n}(z),
$$

where $z \in D$ is uniquely determined unless $|z|=1$.

It is well known that the state space of $B\left(\mathbb{C}^{2}\right)$ is affinely isomorphic to the unit ball of $\mathbb{R}^{3}$ such that any pure state on $B\left(\mathbb{C}^{2}\right)$ can be identified with an element of $S^{2}$. 
The nonuniqueness of our parametrization of pure states on $B\left(\mathbb{C}^{2}\right)$ by means of elements of the set $D$ is due to the fact that the unit sphere $S^{2}$ and the closed disc $D$ are not homeomorphic. However, as Eq. (28) shows, our parametrization is convenient from a probabilistic viewpoint.

We are prepared now to consider a countably infinite system of TLS which is characterized by the algebra $\stackrel{\mathbb{N}}{\otimes} B\left(\mathbb{C}^{2}\right)$ of observables. We confine ourselves to Bose-Einstein symmetric states, i.e. symmetric states which have the property that (22) holds for any finite-dimensional marginal statistical operator. $\mathbb{N}$

In [1] it is shown that the set of Bose-Einstein symmetric states on $\otimes B\left(\mathbb{C}^{2}\right)$ is a Choquet simplex which is generated (in the weak*-topology) as the closed convex hull of its extreme points which are precisely the homogeneous product states of pure states. Accordingly, for any finite-dimensional reduced statistical operator an integral representation exists.

Theorem 3 [1]. For any Bose-Einstein symmetric state on $\otimes B\left(\mathbb{C}^{2}\right)$ there exists a probability measure $v \in M_{+}^{1}(D)$ such that for any $n \in \mathbb{N}$ the reduced statistical operators $W_{n}$ defined on $\left(\stackrel{n}{\otimes} \mathbb{C}^{2}\right)$, admit a representation as trace class operator valued Bochner integrals in terms of $n$-coherent states

$$
W_{n}(v)=\int d v(z) P_{n}(z) .
$$

Moreover, up to the homogeneous product state defined by means of the statistical operator (31) the mixing measure $v$ in (33) is uniquely determined.

The proof is obvious from the results of [1]. Nonuniqueness is an artificial consequence of our parametrization. For the fact that the integrals can be considered as trace class operator valued Bochner integrals cf. [4].

$\mathbb{N}$

Example 3. Assume a Bose-Einstein symmetric state on $\otimes B\left(\mathbb{C}^{2}\right)$ is characterized by a mixing measure $v \in M_{+}^{1}(D)$ which is invariant under rotations, then $W_{n}(v)$ is diagonal in the number states, $n \in \mathbb{N}$,

$$
W_{n}(v)=\int d v(z) P_{n}(z)=\sum_{k=0}^{n}\left\{\int d v(z) B_{n,|z|^{2}}(k)\right\}\left\langle\phi_{n}(k), \cdot\right\rangle \phi_{n}(k) .
$$

In fact, under the assumption of rotation invariance we have for all $z \in D$,

$$
\int_{0}^{2 \pi} \frac{d \alpha}{2 \pi} P_{n}(z)=\sum_{k=0}^{n} B_{n,\left.|z|\right|^{2}}(k)\left\langle\phi_{n}(k), \cdot\right\rangle \phi_{n}(k) .
$$

\section{The Poisson Limit}

In analogy to the situation analyzed in Theorem 2 we consider an array of TLS. This array consists of the sequence of rows $\left(\otimes B\left(\mathbb{C}^{2}\right), v_{n}\right), n \in \mathbb{N}$, where $v_{n} \in M_{+}^{1}(D)$ is the mixing measure of a Bose-Einstein symmetric state on $\otimes B\left(\mathbb{C}^{2}\right)$. In view of Theorem 3 the array is characterized by the array of reduced statistical operators, $n \in \mathbb{N}, m \in \mathbb{N}$,

$$
W_{m}\left(v_{n}\right)=\int d v_{n}(z) P_{m}(z) .
$$


In [2] it is shown that for any $k, k^{\prime} \in \mathbb{Z}_{+}$and any $z \in \mathbb{C}$,

$$
\lim _{n \rightarrow \infty}\left\langle\phi_{n}(k), P_{n}(z / \sqrt{n}) \phi_{n}\left(k^{\prime}\right)\right\rangle=\left\langle\Phi(k), P(z) \Phi\left(k^{\prime}\right)\right\rangle,
$$

and for any $u, v \in \mathbb{C}$ and any $z \in \mathbb{C}$,

$$
\lim _{n \rightarrow \infty}\left\langle\psi_{n}(u / \sqrt{n}), P_{n}(z / \sqrt{n}) \psi_{n}(v / \sqrt{n})\right\rangle=\langle\Psi(u), P(z) \Psi(v)\rangle
$$

holds where the quantities on the right-hand side are defined in Sect. 1.

From the physical viewpoint this means that the matrix elements of the statistical operator $P_{n}(z / \sqrt{n})$, evaluated by means of $n$-coherent vectors or $n$-number vectors, converge to the matrix elements of the statistical operator $P(z)$ evaluated by means of coherent vectors or number vectors. In the following we show that this convergence of matrix elements extends to mixtures of $n$-coherent states, mixtures of coherent states, respectively. To this end we need a preliminary lemma.

Lemma 1. Assume that the sequence $\mu_{n} \in M_{+}^{1}(\mathbb{C})$ converges weakly to $\mu \in M_{+}^{1}(\mathbb{C})$. Moreover, suppose that the sequence $f_{n} \in C_{b}(\mathbb{C})$ is uniformly bounded, $\left|f_{n}\right| \leqq c_{0}$, and converges uniformly on compact sets to $f \in C_{b}(\mathbb{C})$, then

$$
\lim _{n \rightarrow \infty} \int d \mu_{n}(z) f_{n}(z)=\int d \mu(z) f(z) .
$$

Proof. We have

$$
\begin{aligned}
\left|\int d \mu_{n}(z) f_{n}(z)-\int d \mu(z) f(z)\right| \leqq & \left|\int d \mu_{n}(z) f(z)-\int d \mu(z) f(z)\right| \\
& +\int d \mu_{n}(z)\left|f_{n}(z)-f(z)\right|=I_{n}+I I_{n} .
\end{aligned}
$$

The first term vanishes in the limit $n \rightarrow \infty$ because of the fact that $f \in C_{b}(\mathbb{C})$ and the weak convergence $\mu_{n} \rightarrow \mu$. It remains to show that for any $\varepsilon>0$ there exists an integer $N_{\varepsilon}$ such that for all $n>N_{\varepsilon}$ we have $I I_{n}<\varepsilon$.

As the sequence $\mu_{n} \in M_{+}^{1}(\mathbb{C})$ converges weakly it is uniformly tight. Accordingly, for any $\delta>0$ there exists a compact set $K(\delta) \subset \mathbb{C}$ such that for all $n \in \mathbb{N}$ we have $\mu_{n}(\mathbb{C} \backslash K(\delta))<\delta$. Moreover, by virtue of uniform convergence, for any $\eta>0$ and any compact set $K \subset \mathbb{C}$ there exists an integer $N(\eta, K)$ such that for all $n>N(\eta, K)$,

$$
\sup _{z \in K}\left|f_{n}(z)-f(z)\right|<\eta \text {. }
$$

Let $\varepsilon$ be given and set $N_{\varepsilon}=N\left(\frac{\varepsilon}{2}, K\left(\frac{\varepsilon}{2\left(c_{0}+c\right)}\right)\right)$, where $c$ is chosen such that $|f| \leqq c$. With this choice of $N_{\varepsilon}$ we obtain

$$
\begin{aligned}
I I_{n} & =\int d \mu_{n}(z)\left|f_{n}(z)-f(z)\right| 1_{K}\left(\frac{\varepsilon}{2\left(c_{0}+c\right)}\right)(z)+\int d \mu_{n}(z)\left|f_{n}(z)-f(z)\right| 1_{\mathbb{C} \backslash K\left(\frac{\varepsilon}{2\left(c_{0}+c\right)}\right)}(z) \\
& \leqq \sup _{z \in K\left(\frac{\varepsilon}{2\left(c_{0}+c\right)}\right)}\left|f_{n}(z)-f(z)\right|+\left(c_{0}+c\right) \mu_{n}\left(\mathbb{C} \backslash K\left(\frac{\varepsilon}{2\left(c_{0}+c\right)}\right)\right) \\
& \leqq \frac{\varepsilon}{2}+\left(c_{0}+c\right) \frac{\varepsilon}{2\left(c_{0}+c\right)}=\varepsilon,
\end{aligned}
$$

which ends the proof. 
Theorem 4. Let be given an array $\left(\stackrel{\mathbb{N}}{\otimes} B\left(\mathbb{C}^{2}\right), v_{n}\right), n \in \mathbb{N}$, of TLS, where $v_{n} \in M_{+}^{1}(D)$ is the mixing measure associated with a Bose-Einstein symmetric state on $\otimes B\left(\mathbb{C}^{2}\right)$. Denote by $\mu_{n} \in M_{+}^{1}(\mathbb{C})$ the image of $v_{n} \in M_{+}^{1}(D)$ under the mapping $\tau_{n}: D \rightarrow \mathbb{C}$ which is defined by $\tau_{n}(z)=z \sqrt{n}$. Moreover, assume that the sequence $\mu_{n}, n \in \mathbb{N}$, converges weakly to a probability measure $\mu \in M_{+}^{1}(\mathbb{C})$ then the sequence $W_{n}\left(v_{n}\right)$ converges to the classical state $W(\mu)$ in the sense that i) for all $k, k^{\prime} \in \mathbb{Z}_{+}$,

$$
\lim _{n \rightarrow \infty}\left\langle\phi_{n}(k), W_{n}\left(v_{n}\right) \phi_{n}\left(k^{\prime}\right)\right\rangle=\left\langle\Phi(k), W(\mu) \Phi\left(k^{\prime}\right)\right\rangle
$$

and ii) for all $u, v \in \mathbb{C}$,

$$
\lim _{n \rightarrow \infty}\left\langle\psi_{n}(u / \sqrt{n}), W_{n}\left(v_{n}\right) \psi_{n}(v / \sqrt{n})\right\rangle=\langle\Psi(u), W(\mu) \Psi(v)\rangle
$$

hold.

Proof. Using the notation introduced in the theorem we have for all $n \in \mathbb{N}$,

$$
W_{n}\left(v_{n}\right)=\int d \mu_{n}(z) P_{n}(z / \sqrt{n}) .
$$

For part i) we set

$$
\begin{aligned}
f_{n}^{(i)}(z) & =\left\langle\phi_{n}(k), \psi_{n}(z / \sqrt{n})\right\rangle\left\langle\psi_{n}(z / \sqrt{n}), \phi_{n}\left(k^{\prime}\right)\right\rangle \\
& =\left\{B_{n,|z|^{2} / n}(k) B_{n,|z|^{2} / n}\left(k^{\prime}\right)\right\}^{1 / 2} \exp \left(i \alpha\left(k-k^{\prime}\right)\right),
\end{aligned}
$$

and for part ii) we set

$$
\begin{aligned}
f_{n}^{(i i)}(z)= & \left\langle\psi_{n}(u / \sqrt{n}), \psi_{n}(z / \sqrt{n})\right\rangle\left\langle\psi_{n}(z / \sqrt{n}), \psi_{n}(v / \sqrt{n})\right\rangle \\
= & {\left[\frac{\bar{u} z}{n}+\left\{\left(1-\frac{|u|^{2}}{n}\right)\left(1-\frac{|z|^{2}}{n}\right)\right\}^{1 / 2}\right]^{n} } \\
& \times\left[\frac{\bar{z} v}{n}+\left\{\left(1-\frac{|z|^{2}}{n}\right)\left(1-\frac{|v|^{2}}{n}\right)\right\}^{1 / 2}\right]^{n} .
\end{aligned}
$$

Obviously,

$$
\lim _{n \rightarrow \infty} f_{n}^{(i)}(z)=\left\{\pi_{\lambda}(k) \pi_{\lambda}\left(k^{\prime}\right)\right\}^{1 / 2} \exp \left(i \alpha\left(k-k^{\prime}\right)\right),
$$

where we set $\lambda=|z|^{2}$ and

$$
\lim _{n \rightarrow \infty} f_{n}^{(i i)}(z)=\exp \left(-\frac{|u|^{2}+|v|^{2}}{2}-|z|^{2}+\bar{u} z+\bar{z} v\right) .
$$

Moreover, in both cases the assumptions of Lemma 1 are fulfilled such that the convergence of the matrix elements is a consequence of Lemma 1. This ends the proof of Theorem 4.

Example 4. Assume that the mixing measure $v_{n}$ in any row of the array

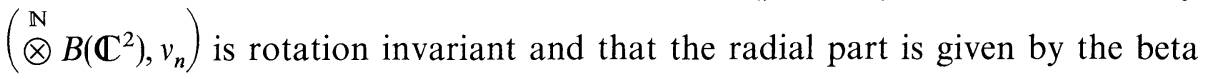
distributions of Example 2,

$$
W_{m}\left(v_{n}\right)=\int \frac{d \alpha}{\pi} d|z||z|(d(n)-1)\left(1-|z|^{2}\right)^{d(n)-2} P_{m}(z) .
$$


From Example 3 we obtain

$$
W_{m}\left(v_{n}\right)=\sum_{k=0}^{m}\left(\begin{array}{c}
d(n)+m-1 \\
m
\end{array}\right)^{-1}\left(\begin{array}{c}
d(n)+m-k-2 \\
m-k
\end{array}\right)\left\langle\phi_{m}(k), \cdot\right\rangle \phi_{m}(k) .
$$

Under these conditions the sequence $\mu_{n}$ associated with $v_{n}$ converges to a rotation invariant probability measure on $\mathbb{C}$ which is defined by the property that the radial part is an exponential distribution with mean $\bar{n}$. In the sense of the convergence which is explained in Theorem 4 we therefore obtain $W_{n}\left(v_{n}\right) \rightarrow W(\mu)$, where $W(\mu)$ is given by

$$
W(\mu)=\int \frac{d^{2} z}{\pi}(\bar{n})^{-1} \exp \left(-|z|^{2} / \bar{n}\right) P(z)=\sum_{k=0}^{\infty} \frac{1}{1+\bar{n}}\left(\frac{\bar{n}}{1+\bar{n}}\right)^{k}\langle\Phi(k), \cdot\rangle \Phi(k) .
$$

\section{Remarks}

A preliminary version of Theorem 4 has been announced in [10]. Due to the fact that we want to formulate this theorem in the general setting proposed in [2] we changed the formulation and the strategy of the proof.

In the context of the classical de Finetti theorem it is well known that sequences of interchangeable random variables are not necessarily infinitely-extendible such that, in this case, no integral representation exists (there exists, however, a representation as compound hypergeometric distribution). This entails, on the level of the Poisson limit of de Finetti's theorem, that not all elements of $M_{+}^{1}\left(\mathbb{Z}_{+}\right)$ admit an integral representation in terms of Poisson distributions.

In the canonical formulation of quantum theory the spectral theorem guarantees, given the state, that in any abelian subalgebra a formulation of expectations by means of a uniquely determined classical probability measure exists. As far as the abelian subalgebra generated by the number operator is concerned this implies that expectations are canonically determined by an element of $M_{+}^{1}\left(\mathbb{Z}_{+}\right)$. This classical formulation of expectations, however, does not entail that an integral representation for the probability distribution on $\mathbb{Z}_{+}$exists.

The fact that the phenomenon of nonrepresentability is already present in the abelian subalgebra generated by the number operator shows that the $P$-representation (cf. e.g. [11]) of nonclassical states is based on concepts (distributions, signed measures) which, in the canonical formulation of quantum theory, have no physical meaning.

These so-called "negative probabilities" are not the result of some inherent and typical quantum behaviour, transcending classical concepts, but rather indicate lack of sufficient extendibility properties of the Bose-Einstein symmetric states on the array of quanta under consideration. As the extendibility properties are connected with the correlation structure of the array we conclude that the nonclassical properties of nonclassical states are the consequence of specific correlations (e.g. strictly negative correlations).

For the investigation of the properties of nonclassical states an analysis of the precise interrelation between the extendibility properties and the correlation structure is therefore more interesting than the construction of objects which are devoid of any physical meaning in classical and in quantum probability. 
We conclude with a remark concerning the mixing measure of the harmonic oscillator in thermal equilibrium (Example 4). The derivation of the mixing measure given here shows that the gaussian distribution is not a consequence of the CLT but rather a consequence of Bose-Einstein statistics. The exponential distribution (Maxwell-Boltzmann distribution) is derived from the interchangeable array of Example 2 which is subject to Bose-Einstein statistics.

Acknowledgements. A.B. is very grateful to Prof. L. Accardi for the kind and stimulating hospitality at the Centro Matematico V. Volterra of the Università di Roma II, Tor Vergata, where this work was completed.

\section{References}

1. Hudson, R.L., Moody, G.R.: Locally normal symmetric states and an analogue of de Finetti's theorem. Z. Wahrscheinlichkeitstheorie Verw. Geb. 33, 343-351 (1976)

2. Accardi, L., Bach, A.: The harmonic oscillator as quantum central limit of quantum Bernoulli processes. Probab. Theory Rel. Fields (to appear)

3. Davis, E.B.: Quantum theory of open systems. London: Academic Press 1976

4. Bach, A., Lüxmann-Ellinghaus, U.: The simplex structure of the classical states of the quantum harmonic oscillator. Commun. Math. Phys. 107, 553-560 (1986)

5. Aldous, D.: Exchangeability and related topics. Lecture Notes in Mathematics, Vol. 1117, pp. 1-198. Berlin, Heidelberg, New York: Springer 1985

6. Bach, A.: The concept of indistinguishable particles in classical and quantum physics. Found. Phys. 18, 639-649 (1988)

7. Feller, W.: An introduction to probability theory and its applications, Vol. II. New York: Wiley 1966

8. Benczur, A.: On sequences of equivalent events and the compound Poisson process. Stud. Sci. Math. Hungarica 3, 451-458 (1968)

9. Bothe, W.: Zur Statistik der Hohlraumstrahlung. Z. Phys. 41, 345-351 (1927)

10. Bach, A.: Integral representations by means of coherent states derived from de Finetti's theorem. Europhys. Lett. 4, 383-387 (1987)

11. Klauder, J.R., Sudarshan, E.C.G.: Fundamentals of quantum optics. New York: Benjamin 1968

Communicated by G. Parisi

Received November 1, 1988 